\title{
Article
}

\section{Completion of BCC-algebras}

\section{S. Mehrshad}

Faculty of Sciences, Zabol University of Zabol, Iran.; smehrshad@uoz.ac.ir

Received: 25 March 2020; Accepted: 27 September 2020; Published: 2 October 2020.

Abstract: In this paper, we study some properties of induced topology by a uniform space generated by a family of ideals of a BCC-algebra. Also, by using Cauchy nets we construct a uniform space which is completion of this space.

Keywords: $B C C$-algebra, uniform space, cauchy net, ideal.

MSC: 06B10, 03G10.

\section{Introduction}

I

n 1966, Y. Imai and K. Iséki in [1] introduced a class of algebras of type $(2,0)$ called BCK-algebras which generalizes on one hand the notion of algebra of sets whit the set subtraction as the only fundamental non-nullary operation, on the other hand the notion of implication algebra. K. Iséki posed an interesting problem whether the class of BCK-algebras form a variety. In connection with this problem Y. Komori in [2] introduced a notion of BCC-algebras which is a generalization of notion BCK-algebras and proved that class of all BCC-algebras is not a variety. W. A. Dudek in [3] redefined the notion of BCC-algebras by using a dual form of the ordinary definition. Further study of BCC-algebras was continued [4-6].

In 1937, André Weil in [7] introduced the concept of a uniform space as a generalization of the concept of a metric space in which many non-topological invariants can be defined. The study of quasi uniformities started in 1948 with Nachbin's investigations on uniform preordered spaces. Mehrshad and Kouhestani in [8] introduced a quasi-uniformity on a BCC-algebra by a family of ideals and studied some properties of this structure. Now, in this present work, we consider the set $C$ of all cauchy nets on BCC-algebras $X$ and define a congruence relation $\sim$ on this set. Then we consider the quotient BCC-algebra $\mathcal{C}=\frac{\mathcal{C}}{\sim}$ and prove that $\mathcal{C}$ is a BCC-algebra. We construct a uniformity on $\mathcal{C}$ and show that this uniformity is a completion of uniform space on $X$ induced by a family of ideals of $X$.

\section{Preliminary}

\section{BCC-algebras}

A BCC-algebra is a non empty set $X$ with a constant 0 and a binary operation $*$ satisfying the following axioms, for all $x, y, z \in X$ :

(1) $((x * y) *(z * y)) *(x * z)=0$,

(2) $0 * x=0$,

(3) $x * 0=x$

(4) $x * y=0$ and $y * x=0$ imply $x=y$.

A non empty subset $S$ of BCC-algebra $X$ is called subalgebra of $X$ if it is closed under BCC-operation. For a BCC-algebra $X$, we denote $x \wedge y=y *(y * x)$ for all $x, y \in X$. On any BCC-algebra $X$ one can define the natural order $\leq$ putting

$$
x \leq y \Leftrightarrow x * y=0 .
$$

It is not difficult to verify that this order is partial and 0 is its smallest element. In BCC-algebra $X$, following hold: for any $x, y, z \in X$

(5) $(x * y) *(z * y) \leq x * z$,

(6) $x \leq y$ implies $x * z \leq y * z$ and $z * y \leq z * x$, 
(7) $x \wedge y \leq x, y$

(8) $x * y \leq x$

(9) $(x * y) * z \leq x *(y * z)$

(10) $x * x=0$,

(11) $(x * y) * x=0[$ see, [6]].

Definition 1. [9] Let $X$ be a BCC-algebra and $\varnothing \neq I \subseteq X$. I is called an ideal of $X$ if it satisfies the following conditions:

(12) $0 \in I$,

(13) $x * y \in I$ and $y \in I$ imply $x \in I$.

If $I$ is an ideal in BCC-algebra of $X$, then $I$ is a subalgebra. Moreover, if $x \in I$ and $y \leq x$, then $y \in I$. An ideal $I$ is said to be regular ideal if the relation

$$
x \equiv{ }^{I} y \Longleftrightarrow x * y, y * x \in I
$$

is a congruence relation. In this case we denote $x / I=\left\{y: x \equiv^{I} y\right\}$ and $X / I=\{x / I: x \in X\} . X / I$ is a BCC-algebra by $x / I * y / I=(x * y) / I$.

\section{Uniform and quasi uniform space}

Let $A$ be a non-empty set and $\varnothing \neq \mathcal{F} \subseteq P(A)$. Then $\mathcal{F}$ is called a filter on $P(A)$, if for each $F_{1}, F_{2} \in \mathcal{F}$ :

(i) $F_{1} \in \mathcal{F}$ and $F_{1} \subseteq F$ imply $F \in \mathcal{F}$,

(ii) $F_{1} \cap F_{2} \in \mathcal{F}$,

(iii) $\varnothing \notin \mathcal{F}$.

A subset $\mathcal{B}$ of a filter $\mathcal{F}$ on $A$ is a base of $\mathcal{F}$ iff, every set of $\mathcal{F}$ contains a set of $\mathcal{B}$. If $\mathcal{F}$ is a family of nonempty subsets of $A$, then we denote generated filter by $\mathcal{F}$ with $\operatorname{fil}(\mathcal{F})$.

A quasi-uniformity on a set $A$ is a filter $Q$ on $P(X \times X)$ such that

(i) $\triangle=\{(x, x) \in A \times A: x \in A\} \subseteq q$, for each $q \in Q$,

(ii) For each $q \in Q$, there is a $p \in Q$ such that $p \circ p \subseteq q$ where

$$
p \circ p=\{(x, y) \in A \times A: \exists z \in A \text { s.t }(x, z),(z, y) \in p\} .
$$

The pair $(A, Q)$ is called a quasi-uniform space. If $Q$ is a quasi-uniformity on a set $A$, then $q^{-1}=$ $\left\{q^{-1}: q \in Q\right\}$ is also a quasi-uniformity on $A$ called the conjugate of $Q$. It is well-known that if a quasi-uniformity satisfies condition: $q \in Q$ implies $q^{-1} \in Q$, then $Q$ is a uniformity. Also $Q$ is a uniformity on A provided

$$
\forall q \in Q \exists p \in Q \text { s.t } p^{-1} \circ p \subseteq q .
$$

Furthermore, $Q^{*}=Q \vee Q^{-1}$ is a uniformity on $A$. A subfamily $\mathcal{C}$ of quasi-uniformity $Q$ is said to be a base for $Q$ iff, each $q \in Q$ contains some member of $\mathcal{C}$. The topology $T(Q)=\{G \subseteq X: \forall x \in G \exists q \in Q$ s.t $q(x) \subseteq G\}$ is called the topology induced by the quasi-uniformity $Q$ [ See, [10]].

\section{Main results}

Let $X$ be a $B C C$-algebra and $\eta$ be an arbitrary family of ideals of $X$ which is closed under intersection.

Theorem 1. [8] Let $X$ be a BCC-algebra. The set $\mathcal{I}=\left\{I_{L}: I \in \eta\right\}$ is a base for a quasi uniformity $\mathcal{U}$ on $X$, where $I_{L}=\{(x, y) \in X \times X: y * x \in I\}$.

Lemma 1. [8] Let I be a regular ideal of BCC-algebra $X$. Define $I_{L}^{-1}=\left\{(x, y) \in X \times X:(y, x) \in I_{L}\right\}$ and $I_{L}^{\star}=$ $I_{L} \cap I_{L}^{-1}$. Then following holds:

(i) $I_{L}^{-1}=\{(x, y) \in X \times X: x * y \in I\}$ ，

(ii) $I_{L}^{-1}(x)=\{y \in X: x * y \in I\}$,

(iii) $I_{L}^{-1}(0)=X$,

(iv) $I_{L}^{\star}=\left\{(x, y) \in X \times X: x \equiv^{I} y\right\}$ ， 
(v) $I_{L}^{\star}(x)=\left\{y \in X: x \equiv^{I} y\right\}=x / I$,

(vi) if $x \in I$, then $I_{L}^{\star}(x)=I$.

Theorem 2. [8] Let $\mathcal{U}^{\star}=\left\{U \subseteq X \times X: \exists I \in \eta I_{L}^{\star} \subseteq U\right\}$. Then the pair $\left(X, \mathcal{U}^{\star}\right)$ is a uniform space. Moreover, $\left(X, T\left(\mathcal{U}^{\star}\right)\right)$ is a topological BCC-algebra, where $T\left(\mathcal{U}^{\star}\right)=\left\{G \subseteq X: \forall x \in G \exists I \in \eta I_{L}^{\star}(x) \subseteq G\right\}$ is the induced topology by $\mathcal{U}^{\star}$ on $X$.

$$
\text { Let } J=\bigcap_{I \in \eta} I \text {. Then } \mathcal{U}^{\star}=\left\{U \subseteq X \times X: J_{L}^{\star} \subseteq U\right\} \text { and } \tau_{J}=\left\{G \subseteq X: \forall x \in G J_{L}^{\star}(x) \subseteq G\right\} .
$$

Proposition 1. $T\left(\mathcal{U}^{\star}\right)=\tau_{J}$, where $J=\bigcap_{I \in \eta} I$.

Proof. Let $x \in G \in T\left(\mathcal{U}^{\star}\right)$. Then there exists $I \in \eta$ such that $I_{L}^{\star}(x) \subseteq G$. Since for any $I \in \eta J \subseteq I$, we get $J_{L}^{\star} \subseteq I_{L}^{\star}$. Hence $J_{L}^{\star}(x) \subseteq I_{L}^{\star}(x) \subseteq G$ and so $G \in \tau_{\text {J }}$. Thus $T\left(\mathcal{U}^{\star}\right) \subseteq \tau_{\text {J }}$. Conversely, let $x \in G \in \tau_{\text {J }}$. Then $J_{L}^{\star}(x) \subseteq G$. Since $\eta$ is closed under intersection, $J \in \eta$ and so $J_{L}^{\star} \in \mathcal{U}^{\star}$. Hence $G \in T\left(\mathcal{U}^{\star}\right)$. Therefore $\tau_{J} \subseteq T\left(\mathcal{U}^{\star}\right)$.

Definition 2. [11]

(i) A poset $(D, \leq)$ is called an upward directed set if for any $i, j \in D$ there exists $k \in D$ such that $i \leq k$ and $j \leq k$.

(ii) Let $(D, \leq)$ be an upward directed set and $X$ be a BCC-algebra. The mapping $x: D \rightarrow X$ is called a net in $X$ and denoted by $\left\{x_{i}\right\}_{i \in D}$.

Definition 3. Let $\left\{x_{i}\right\}_{i \in D}$ be a net in topological space $\left(X, \tau_{J}\right)$. Then

(i) $\left\{x_{i}\right\}_{i \in D}$ is called converges to $x \in X$ if for any neighborhood $G$ of $x$ there exists $i_{0} \in D$ such that $x_{i} \in G$ for any $i \geq i_{0}$. In this case we write $x_{i} \rightarrow x$.

(ii) $\left\{x_{i}\right\}_{i \in D}$ is called Cauchy if there exists $i_{0} \in D$ such that $\frac{x_{i}}{J}=\frac{x_{j}}{J}$ for any $i, j \geq i_{0}$.

Proposition 2. Let $\left\{x_{i}\right\}_{i \in D}$ and $\left\{y_{i}\right\}_{i \in D}$ be two nets in $\left(X, \tau_{J}\right)$. Then

(i) If $x, y \in X, x_{i} \rightarrow x$ and $y_{i} \rightarrow y$, then $x_{i} * y_{i} \rightarrow x * y$.

(ii) Each convergent net in $X$ is a cauchy net.

Proof. (i) Let $x * y \in G \in \tau_{\mathrm{J}}$. Then $J_{L}^{\star}(x * y) \subseteq G$. Since $x_{i} \rightarrow x$ and $J_{L}^{\star}(x)$ is a neighbohood of $x$, there exists $i_{0} \in D$ such that $x_{i} \in J_{L}^{\star}(x)$ for any $i \geq i_{0}$. Similarly, there exists $i_{1} \in D$ such that $y_{i} \in I_{L}^{\star}(y)$ for any $i \geq i_{1}$. Since $D$ is an upward directed set, there exists $i_{2} \in D$ such that $i_{0}, i_{1} \leq i_{2}$. Hence by Lemma (1) $x_{i} * y_{i} \in J_{L}^{\star}(x) * J_{L}^{\star}(y)=\frac{x}{J} * \frac{y}{J}=\frac{x * y}{J}=J_{L}^{\star}(x * y) \subseteq G$ for any $i \geq i_{2}$ and so $x_{i} * y_{i} \rightarrow x * y$.

(ii) Let $\left\{x_{i}\right\}_{i \in D}$ be a net in $X$ and $x_{i} \rightarrow x \in X$. Since $J_{L}^{\star}(x)$ is a neighborhood of $x$, there exists $i_{0} \in D$ such that $x_{i} \in J_{L}^{\star}(x)$ for any $i \geq i_{0}$. Hence $x_{i} \equiv^{J} x$ and $x_{j} \equiv J x$ for any $i, j \geq i_{0}$ and so $x_{i} \equiv^{J} x_{j}$ for any $i, j \geq i_{0}$. Therefore $\frac{x_{i}}{J}=\frac{x_{j}}{J}$ for any $i, j \geq i_{0}$. Thus $\left\{x_{i}\right\}_{i \in D}$ is a cauchy net in $X$.

Definition 4. [11] Let $(A, Q)$ be a uniform space.

(i) A net $\left\{x_{i}\right\}_{i \in D}$ in $A$ is said to converge to a point $x \in A$ if for each $q \in Q$ there exists $i_{0} \in D$ such that $\left(x_{i}, x\right) \in q$ for any $i \geq i_{0}$.

(ii) A net $\left\{x_{i}\right\}_{i \in D}$ in $A$ is said to be a Cauchy net if for each $q \in Q$ there exists $i_{0} \in D$ such that $\left(x_{i}, x_{j}\right) \in q$ for any $i, j \geq i_{0}$.

Let $C$ be the set of all Cauchy sequence in $\left(X, \mathcal{U}^{\star}\right)$. define a binary relation on $C$ in the following way. For each $\left\{x_{i}\right\}_{i \in D},\left\{y_{j}\right\}_{j \in D} \in C,\left\{x_{i}\right\}_{i \in D} \sim\left\{y_{j}\right\}_{j \in D}$ if and only if for all $U \in \mathcal{U}^{\star}$ there exist $i_{0}, j_{0} \in D$ such that $\left(x_{i}, y_{j}\right) \in G$ for any $i \geq i_{0}$ and $j \geq j_{0}$.

Theorem 3. The relation $\sim$ is a congruence relation on $C$.

Proof. Since $\left(X, \mathcal{U}^{\star}\right)$ is a uniform space, $\triangle \subseteq U$ for any $U \in \mathcal{U}^{\star}$. Hence $\left(x_{i}, x_{i}\right) \in U$ for any $i \in D$ and so $\left\{x_{i}\right\}_{i \in D} \sim\left\{x_{i}\right\}_{i \in D}$. Let $\left\{x_{i}\right\}_{i \in D} \sim\left\{y_{j}\right\}_{j \in D}$. Then for all $U \in \mathcal{U}^{\star}$ there exist $i_{0}, j_{0} \in D$ such that $\left(x_{i}, y_{j}\right) \in U$ for any $i \geq i_{0}$ and $j \geq j_{0}$. Since $U \in \mathcal{U}^{\star}, U^{-1} \in \mathcal{U}^{\star}$. By definition of $U^{-1}$ we have $\left(y_{j}, x_{i}\right) \in \mathcal{U}^{-1}$ for any $i \geq i_{0}$ 
and $j \geq j_{0}$. Hence $\left\{y_{j}\right\}_{j \in D} \sim\left\{x_{i}\right\}_{i \in D}$. Let $\left\{x_{i}\right\}_{i \in D} \sim\left\{y_{j}\right\}_{j \in D}$ and $\left\{y_{j}\right\}_{j \in D} \sim\left\{z_{i}\right\}_{i \in D}$. Let $U \in \mathcal{U}^{\star}$. There exists $V \in \mathcal{U}^{\star}$ such that $V \circ V \subseteq U$. Since $\left\{x_{i}\right\}_{i \in D} \sim\left\{y_{j}\right\}_{j \in D}$, there exist $i_{0}, j_{0} \in D$ such that $\left(x_{i}, y_{j}\right) \in V$ for any $i \geq i_{0}, j \geq j_{0}$. Similarly, there exist $k_{0}, l_{0} \in D$ such that $\left(y_{j}, z_{k}\right) \in V$ for any $j \geq l_{0}, k \geq k_{0}$. Since $D$ is an upward directed set, there exsits $n \in D$ such that $j_{0}, l_{0} \leq n$. If $j \geq n$, then $\left(x_{i}, y_{j}\right) \in V$ and $\left(y_{j}, z_{k}\right) \in V$ for any $i \geq i_{0}$ and $k \geq k_{0}$. Hence $\left(x_{i}, z_{k}\right) \in V \circ V \subseteq U$ for any $i \geq i_{0}$ and $k \geq k_{0}$ and so $\left\{x_{i}\right\}_{i \in D} \sim\left\{z_{k}\right\}_{k \in D}$. Thus $\sim$ is an equivalence relation on $C$. Finally, we show that $\sim$ is congruence. Let $I \in \eta,\left\{x_{i}\right\}_{i \in D} \sim\left\{y_{j}\right\}_{j \in D}$ and $\left\{z_{k}\right\}_{k \in D} \sim\left\{w_{l}\right\}_{l \in D}$. Hence there exist $i_{0}, j_{0}, k_{0}$ and $l_{0} \in D$ such that $\left(x_{i}, y_{j}\right) \in I_{L}^{\star}$ for any $i \geq i_{0}, j \geq j_{0}$ and $\left(z_{k}, w_{l}\right) \in I_{L}^{\star}$ for any $k \geq k_{0}$ and $l \geq i_{0}$. Let $i \geq i_{0}, j \geq j_{0}$ and $k \geq k_{0}$. Then $y_{j} \in I_{L}^{\star}\left(x_{i}\right)$ and $z_{k} \in L_{L}^{\star}\left(z_{k}\right)$. Thus $y_{j} * z_{k} \in I_{L}\left(x_{i}\right) * z_{k} \subseteq I_{L}^{\star}\left(x_{i}\right) * I_{L}^{\star}\left(z_{k}\right)=I_{L}^{\star}\left(x_{i} * z_{k}\right)$ and so $\left(x_{i} * z_{k}, y_{j} * z_{k}\right) \in I_{L}^{\star}$. Similarly, If $j \geq j_{0}, k \geq k_{0}$ and $l \geq l_{0}$, then $\left(y_{j} * z_{k}, y_{j} * w_{l}\right) \in I_{L}^{\star}$. Thus $\left(x_{i} * y_{j}, z_{k} * w_{l}\right) \in I_{L}^{\star} \circ I_{L}^{\star}$ subseteq $I_{L}^{\star}$ for any $i \geq i_{0}, j \geq j_{0}, k \geq k_{0}$ and $l \geq l_{0}$. Since for each $U \in \mathcal{U}^{\star}$ there exists $I \in \eta$ such that $I_{L}^{\star} \subseteq U,\left(x_{i} * y_{j}, z_{k} * w_{l}\right) \in U$ for $i \geq i_{0}, j \geq j_{0}, k \geq k_{0}$ and $l \geq l_{0}$. Hence $\sim$ is a congruence relation on $C$.

Let $\mathcal{C}=\frac{\mathcal{C}}{\sim}$. Define a binary operation on $\mathcal{C}$ as follow:

$$
*: \mathcal{C} \times \mathcal{C} \rightarrow \mathcal{C} \quad\left(\frac{\left\{x_{i}\right\}_{i \in D}}{\sim}, \frac{\left\{y_{j}\right\}_{j \in D}}{\sim}\right) \rightarrow \frac{\left\{x_{i} * y_{j}\right\}_{i, j \in D}}{\sim} .
$$

Theorem 4. $\left(\mathcal{C}, *, \frac{\{0\}_{i \in D}}{\sim}\right)$ is a BCC-algebra.

Proof. The proof is clear.

Let $\mathcal{V}=\left\{\hat{U}: U \in \mathcal{U}^{\star}\right\}$ where,

$$
\hat{U}=\left\{\left(\frac{\left\{x_{i}\right\}_{i \in D}}{\sim}, \frac{\left\{y_{j}\right\}_{j \in D}}{\sim}\right) \in \mathcal{C} \times \mathcal{C}: \exists i_{0}, j_{0} \in D: \forall i \geq i_{0}, j \geq j_{0},\left(x_{i}, y_{j}\right) \in U\right\} .
$$

Theorem 5. The pair $(\mathcal{C}, \mathcal{V})$ is a uniform space.

Proof. Let $\hat{U} \in \mathcal{V}$ and $\frac{\left\{x_{i}\right\}_{i \in D}}{\sim} \in \mathcal{C}$. Since $\left\{x_{i}\right\}_{i \in D} \sim\left\{x_{i}\right\}_{i \in D}$, there exists $i_{0} \in D$ such that $\left(x_{i}, x_{i}\right) \in U$ for any $i \geq i_{0}$. Hence $\left(\frac{\left\{x_{i}\right\}_{i \in D}}{\sim}, \frac{\left\{x_{i}\right\}_{i \in D}}{\sim}\right) \in \hat{U}$. Since $\frac{\left\{x_{i}\right\}_{i \in D}}{\sim} \in \mathcal{C}$ is arbitrary, we get $\triangle \subseteq \hat{U}$. Let $\hat{U} \in \mathcal{V}$. Then $U \in \mathcal{U}^{\star}$ and so $\mathcal{U}^{-1} \in \mathcal{U}^{\star}$. Hence $\widehat{U^{-1}} \in \mathcal{V}$. We show that $\widehat{U^{-1}}=(\hat{U})^{-1}$. Let $\left(\frac{\left\{x_{i}\right\}_{i \in D}}{\sim}, \frac{\left\{y_{j}\right\}_{j \in D}}{\sim}\right) \in(\hat{U})^{-1}$. Then $\left(\frac{\left\{y_{j}\right\}_{j \in D}}{\sim}, \frac{\left\{x_{i}\right\}_{j \in D}}{\sim}\right) \in \hat{U}$. Hence there exist $i_{0}, j_{0} \in D$ such that $\left(y_{j}, x_{i}\right) \in U$ for any $i \geq i_{0}$ and $j \geq j_{0}$ and so $\left(x_{i}, y_{j}\right) \in U^{-1}$ for any $i \geq i_{0}$ and $j \geq j_{0}$. Therefore $\left(\frac{\left\{x_{i}\right\}_{i \in D}}{\sim}, \frac{\left\{y_{j}\right\}_{i \in D}}{\sim}\right) \in \widehat{U^{-1}}$ and hence $(\hat{U})^{-1} \subseteq \widehat{U^{-1}}$. Similarly, we have $\widehat{U^{-1}} \subseteq(\hat{U})^{-1}$. Thus $(\hat{U})^{-1} \in \mathcal{V}$ for any $\hat{U} \in \mathcal{V}$. Let $\hat{U} \in \mathcal{V}$. Then $U \in \mathcal{U}^{\star}$. There exists $V \in \mathcal{U}^{\star}$ such that $V \circ V \in \mathcal{U}$. We claim that $\hat{V} \circ \hat{V} \subseteq \hat{U}$. Let $\left(\frac{\left\{x_{i}\right\}_{i \in D}}{\sim}, \frac{\left\{z_{k}\right\}_{k \in D}}{\sim}\right) \in \hat{V} \circ \hat{V}$. There exists $\frac{\left\{x_{i}\right\}_{i \in D}}{\sim} \in \mathcal{C}$ such that $\left(\frac{\left\{x_{i}\right\}_{i \in D}}{\sim}, \frac{\left\{y_{j}\right\}_{j \in D}}{\sim}\right) \in \hat{V}$ and $\left(\frac{\left\{y_{j}\right\}_{j \in D}}{\sim}, \frac{\left\{z_{k}\right\}_{k \in D}}{\sim}\right) \in \hat{V}$. Hence there exist $i_{0}, j_{0}, k_{0}$ and $l_{0} \in D$ such that $\left(x_{i}, y_{j}\right) \in V$ for any $i \geq i_{0}, j \geq j_{0}$ and $\left(y_{j}, z_{k}\right) \in V$ for any $j \geq l_{0}, k \geq k_{0}$. Since $D$ is an upward direcred set, there exists $n \in D$ such that $n \geq j_{0}, l_{0}$. If $j \geq n$, then $\left(x_{i}, y_{j}\right) \in V$ and $\left(y_{j}, z_{k}\right) \in V$ for any $i \geq i_{0}, k \geq k_{0}$. Hence $\left(x_{i}, z_{k}\right) \in V \circ V \subseteq U$ for any $i \geq i_{0}, k \geq k_{0}$ and so $\left(\frac{\left\{x_{i}\right\}_{i \in D}}{\sim}, \frac{\left\{z_{k}\right\}_{k \in D}}{\sim}\right) \in \hat{U}$. Let $\hat{U}, \hat{V} \in \mathcal{V}$. Then $U, V \in \mathcal{U}^{\star}$ and so $U \cap \in V \in \mathcal{U}^{\star}$. Hence $\widehat{U \cap V} \in \mathcal{V}$. We show that $\widehat{U \cap V}=\hat{U} \cap \hat{V}$. Let $\left(\frac{\left\{x_{i}\right\}_{i \in D}}{\sim}, \frac{\left\{y_{j}\right\}_{j \in D}}{\sim}\right) \in \hat{U} \cap \hat{V}$. Then $\left(\frac{\left\{x_{i}\right\}_{i \in D}}{\sim}, \frac{\left\{y_{j}\right\}_{j \in D}}{\sim}\right) \in \hat{U}$ and $\left(\frac{\left\{x_{i}\right\}_{i \in D}}{\sim}, \frac{\left\{y_{j}\right\}_{j \in D}}{\sim}\right) \in \hat{V}$. There exist $i_{0}, j_{0}, i_{1}$ and $j_{1} \in D$ such that $\left(x_{i}, y_{j}\right) \in U$ for any $i \geq i_{0}, j \geq j_{0}$ and $\left(x_{i}, y_{j}\right) \in V$ for any $i \geq i_{1}, j \geq j_{1}$. There exist $i_{2}, j_{2} \in D$ such that $i_{0}, i_{1} \leq i_{2}$ and $j_{0}, j_{1} \leq j_{2}$. Hence $\left(x_{i}, y_{j}\right) \in U$ and $\left(x_{i}, y_{j}\right) \in V$ for any $i \geq i_{2}$ and $j \geq j_{2}$ and so $\left(x_{i}, y_{j}\right) \in U \cap V$ for any $i \geq i_{2}$ and $j \geq j_{2}$. Hence $\left(\frac{\left\{x_{i}\right\}_{i \in D}}{\sim}, \frac{\left\{y_{j}\right\}_{j \in D}}{\sim}\right) \in \widehat{U \cap V}$ and so $\hat{U} \cap \hat{V} \subseteq \widehat{U \cap V}$. Similarly, we can show that $\widehat{U \cap V} \subseteq \hat{U} \cap \hat{V}$. Finally, 
let $\hat{U} \in \mathcal{V}$ and $\hat{U} \subseteq \widetilde{V} \subseteq \mathcal{C} \times \mathcal{C}$. We have to show that $\widetilde{V} \in \mathcal{V}$. Let $(x, y) \in U \in \mathcal{U}^{\star}$. Then $\left(\frac{\{x\}_{i \in D}}{\sim}, \frac{\{y\}_{j \in D}}{\sim}\right) \in \hat{U}$ and so $\left(\frac{\{x\}_{i \in D}}{\sim}, \frac{\{y\}_{j \in D}}{\sim}\right) \in \widetilde{V}$. Thus $(x, y) \in V$ and so $U \subseteq V$. Hence $V \in \mathcal{U}^{\star}$ and so $\widetilde{V} \in \mathcal{V}$.

Theorem 6. $(\mathcal{C}, *, T(\mathcal{V}))$ is a topological BCC-algebra where,

$$
T(\mathcal{V})=\left\{G \in \mathcal{C}: \forall \frac{\{x\}_{i \in D}}{\sim} \exists \hat{U} \in \mathcal{V} \text { s.t. } \hat{U}\left(\frac{\{x\}_{i \in D}}{\sim}\right) \subseteq G\right\} .
$$

Proof. Let $\frac{\left\{x_{i}\right\}_{i \in D}}{\sim} * \frac{\left\{y_{j}\right\}_{j \in D}}{\sim} \in G \in T(\mathcal{V})$. Then there exists $U \in \mathcal{U}^{\star}$ such that $\hat{U}\left(\frac{\left\{x_{i} * y_{j}\right\}_{i, j \in D}}{\sim}\right) \subseteq G$. Since $U \in \mathcal{U}^{\star}$, there exists $I \in \eta$ such that $I_{L}^{\star} \subseteq U$. Clearly, $\widehat{I}_{L}^{\star}\left(\frac{\left\{x_{i} * y_{j}\right\}_{i, j \in D}}{\sim}\right) \subseteq \hat{U}\left(\frac{\left\{x_{i} * y_{j}\right\}_{i, j \in D}}{\sim}\right.$. We claim that $\widehat{I_{L}^{\star}}\left(\frac{\left\{x_{i}\right\}_{i \in D}}{\sim}\right) * \widehat{I}_{L}^{\star}\left(\frac{\left\{y_{j}\right\}_{j \in D}}{\sim}\right) \subseteq \widehat{I_{L}^{\star}}\left(\frac{\left\{x_{i} * y_{j}\right\}_{i, j \in D}}{\sim}\right)$. Let $\frac{\left\{a_{k}\right\}_{k \in D}}{\sim} \in \widehat{I}_{L}^{\star}\left(\frac{\left\{x_{i}\right\}_{i \in D}}{\sim}\right)$ and $\frac{\left\{b_{l}\right\}_{l \in D}}{\sim} \in \widehat{I_{L}^{\star}}\left(\frac{\left\{y_{j}\right\}_{j \in D}}{\sim}\right)$. Then $\left(\frac{\left\{x_{i}\right\}_{i \in D}}{\sim}, \frac{\left\{a_{k}\right\}_{k \in D}}{\sim}\right) \in \widehat{I}_{L}^{\star}$ and $\left(\frac{\left\{y_{j}\right\}_{j \in D}}{\sim}, \frac{\left\{b_{l}\right\}_{l \in D}}{\sim}\right) \in \widehat{I}_{L}^{\star}$. Hence there exist $i_{0}, j_{0}, k_{0}$ and $l_{0} \in D$ such that $\left(x_{i}, a_{k}\right) \in I_{L}^{\star}$ and $\left(y_{j}, b_{l}\right) \in I_{L}^{\star}$ for any $i \geq i_{0}, j \geq j_{0}, k \geq k_{0}$ and $l \geq l_{0}$. Thus $x_{i} \equiv^{I} a_{k}$ and $y_{j} \equiv^{I} b_{l}$ and so $x_{i} * y_{j} \equiv^{I} a_{k} * b_{l}$ for any $i \geq i_{0}, j \geq j_{0}, k \geq k_{0}$ and $l \geq l_{0}$. Therefore $\left(x_{i} * y_{j}, a_{k} * b_{l}\right) \in I_{L}^{\star}$ for any $i \geq i_{0}, j \geq j_{0}, k \geq k_{0}$ and $l \geq l_{0}$ and so $\left(\frac{\left\{x_{i} * y_{j}\right\}_{i, j \in D}}{\sim}, \frac{\left\{a_{k} * b_{l}\right\}_{k, l \in D}}{\sim}\right) \in \widehat{I}_{L}^{\star}$. Hence $\frac{\left\{a_{k} * b_{l}\right\}_{k, l \in D}}{\sim} \in \widehat{I_{L}^{\star}}\left(\frac{\left\{x_{i} * y_{j}\right\}_{i, j \in D}}{\sim}\right)$. Thus $\widehat{I}_{L}^{\star}\left(\frac{\left\{x_{i}\right\}_{i \in D}}{\sim}\right) * \widehat{I}_{L}^{\star}\left(\frac{\left\{y_{j}\right\}_{j \in D}}{\sim}\right) \subseteq$ $\widehat{I_{L}^{\star}}\left(\frac{\left\{x_{i} * y_{j}\right\}_{i, j \in D}}{\sim}\right)$.

Definition 5. [11] The uniform space $(A, Q)$ is complete if each cauchy net in $A$ is convergent.

Definition 6. [11] Let $(A, Q)$ be a uniform space. a uniform space $(\hat{A}, \hat{Q})$ is said to be a completion of $(A, Q)$ if

(i) $(\hat{A}, \hat{Q})$ is a complete uniform space.

(ii) $(A, Q)$ with its topology induced by its uniform structure is homeomorphic to a dense subspace of $(\hat{A}, \hat{Q})$.

Theorem 7. The uniform space $(\mathcal{C}, \mathcal{V})$ is a completion of $\left(X, \mathcal{U}^{\star}\right)$.

Proof. Let $i: X \rightarrow \mathcal{C}$ be defined by $i(x)=\frac{\{x\}_{i \in D}}{\sim}$. Clearly, $i$ is one to one. We show that $i(X)$ is dense in $\mathcal{C}$. Let $\hat{U}\left(\frac{\left\{x_{i}\right\}_{i \in D}}{\sim}\right) \in T(\mathcal{V})$. Then

$$
\begin{aligned}
\hat{U}\left(\frac{\left\{x_{i}\right\}_{i \in D}}{\sim}\right) \cap i(X) & =\left\{i(x):\left(\frac{\left\{x_{i}\right\}_{i \in D}}{\sim}, i(x)\right) \in \hat{U}\right\}, \\
& =\left\{i(x): \exists i_{0} \in D \forall i \geq i_{0} \text { s.t. }\left(x_{i}, x\right) \in U\right\}, \\
& =\left\{i(x): \exists i_{0} \in D \forall i \geq i_{0} \text { s.t. } x \in U\left(x_{i}\right)\right\}, \\
& =\left\{i(x): x \in \bigcup_{i \in D} \bigcap_{i_{0} \leq i} U\left(x_{i}\right)\right\}, \\
& =i(V)
\end{aligned}
$$

where $V=\bigcup_{i \in D} \bigcap_{i_{0} \leq i} U\left(x_{i}\right)$. Hence $\hat{U}\left(\frac{\left\{x_{i}\right\}_{i \in D}}{\sim}\right) \cap i(X) \neq \varnothing$ and so $i(X)$ is dense in $\mathcal{C}$. It is easy to see that $i: X \rightarrow i(X)$ is a homeomorphism. Now we show that the uniform space $(\mathcal{C}, \mathcal{V})$ is complete. Let $\left\{\frac{\left\{x_{i}^{\alpha}\right\}_{i \in D}}{\sim}\right\}_{\alpha \in D}$ be a cauchy net in $\mathcal{C}$. We have to show that it is convergent. Let $U \in \mathcal{U}^{\star}$. Since $\left\{\frac{\left\{x_{i}^{\alpha}\right\}_{i \in D}}{\sim}\right\}_{\alpha \in D}$ is a cauchy net, there exists $\gamma \in D$ such that $\left(\frac{\left\{x_{i}^{\alpha}\right\}_{i \in D}}{\sim}, \frac{\left\{x_{i}^{\beta}\right\}_{i \in D}}{\sim}\right) \in \hat{U}$ for any $\alpha, \beta \geq \gamma$. Hence there exist $\alpha_{0}, \beta_{0} \in D$ such that $\left(x_{i}^{\alpha}, x_{i}^{\beta}\right) \in U$ for any $\alpha \geq \alpha_{0}$ and $\beta \geq \beta_{0}$. We define the net of $\left\{y_{j}\right\}_{j \in D}$ by $y_{j}=x_{i}^{\beta_{0}}$ for any $j \in D$. Clearly, $\left(\frac{\left\{x_{i}^{\alpha}\right\}_{i \in D}}{\sim}, \frac{\left\{y_{j}\right\}_{j \in D}}{\sim}\right) \in \hat{U}$ for any $\alpha \geq \alpha_{0}$. Therefore $\left\{\frac{\left\{x_{i}^{\alpha}\right\}_{i \in D}}{\sim}\right\}_{\alpha \in D}$ is converges to $\frac{\left\{y_{j}\right\}_{j \in D}}{\sim}$. 


\section{Conclusion}

The aim of this paper was to study the concept of completion of a quasi-uniformity on a BCC-algebra. This work can be the basis for further and deeper research of the properties of BCC-algebras.

Conflicts of Interest: "The author declares no conflict of interest."

\section{References}

[1] Imai, Y. \& Iséki, K. (1966). On axioms system of propositional calculi XIV. Proceedings of the Japan Academy, 42, 19-22.

[2] Komori, Y. (1983). The variety generated by BCC-algebras is finitely basded. Reports of the Faculty of Science, Shizuoka University, 17, 13-16.

[3] Dudek, W. A. (1990). On BCC-algebras. Logique et Analyse, 33(129/130), 103-111.

[4] Dudek, W. A. (1999). A new characterization of ideals in BCC-algebras. Novi Sad Journal of Mathematics, 29(1), 139-145.

[5] Dudek, W. A., \& Zhang, X. (1998). On ideals and congruences in BCC-algebras. Czechoslovak Mathematical Journal, 48(1), 21-29.

[6] Dudek, W. A., \& Zhang, X. (1992). On proper BCC-algebras. Bulletin of the Institute of Mathematics, Academia Sinica, 20(2), 137-150.

[7] Weil, A. (1973). sur les espaces a structuure uniforme et sur la topologibgeneral, Gauthier-villars, Paris.

[8] Mehrshad, S., \& Kouhestani, N. (2017). A quasi-uniformity on BCC-algebras. Annals of the University of Craiova-Mathematics and Computer Science Series, 44(1), 64-77.

[9] Hao, J. (1998). Ideal theory of BCC-algebras. Scientiae Mathematicae, 1(3), 373-381.

[10] Fletcher, P. \& Lindgren, W. F.(1982). Quasi-uniform Spaces, Lecture Notes in Pure and Applied Mathematics, Marcel dekker, New York, 77.

[11] Husain, T. (Ed.). (2012). Topology and maps (Vol. 5). Springer Science \& Business Media. 